\title{
Lapurdum
}

Euskal ikerketen aldizkaria | Revue d'études basques |

Revista de estudios vascos | Basque studies review

$15 \mid 2011$

Numéro $X V$

\section{Eduardo Blasco Ferrer. PALEOSARDO - Le radice linguistiche della Sardegna neolitica. De Gruyter, Berlin 2010.}

Jean-Baptiste Orpustan

\section{OpenEdition}

Journals

Édition électronique

URL : http://journals.openedition.org/lapurdum/2335

DOI : 10.4000/lapurdum.2335

ISSN : 1965-0655

Éditeur

IKER

Édition imprimée

Date de publication : 1 octobre 2011

Pagination : 143-148

ISSN : 1273-3830

Référence électronique

Jean-Baptiste Orpustan, «Eduardo Blasco Ferrer. PALEOSARDO - Le radice linguistiche della Sardegna neolitica. De Gruyter, Berlin 2010. », Lapurdum [En ligne], 15 | 2011, mis en ligne le 28 octobre 2014, consulté le 25 avril 2019. URL : http://journals.openedition.org/lapurdum/2335; DOI : 10.4000/ lapurdum.2335 


\section{Compte-rendu :}

\section{Eduardo Blasco Ferrer.}

\section{PALEOSARDO - Le radice}

linguistiche della Sardegna neolitica.

\section{De Gruyter, Berlin 2010.}

Jean-Baptiste ORPUSTAN

IKER (UMR 5478) \& Université Michel de Montaigne - Bordeaux 3

De l'observation et de l'analyse serrée d'un important corpus de noms de lieux (plus de 1.000 noms, microtoponymes pour la plupart) concentrés principalement dans une aire « centro-orientale » de la Sardaigne, les provinces d'Alta Ogliastra, Barbagia et Baronia, remonter jusqu'au temps, il y a plus de 4.000 ans, où l'île utilisait encore une langue apparentée ou même identique au basque ancien tel que, au plan lexical et phono-morphologique, révélé par la toponymie ancienne et datée des territoires de langue basque, et par les travaux d'étymologie et de recontruction menant à un «paléobasque » antérieur aux documentations datées, puis définir les quatre «strates » linguistiques (« paléobasque, ibérique, péri-indoeuropéen, paléoindoeuropéen ») constitutives de ce «paléosarde » des temps néolithiques, peut sembler un itinéraire quasi impossible.

C'est pourtant l'entreprise qu'a menée à bien, au cours d'un travail de longue haleine (plus de 30 titres publiés ou en voie de publication de 1984 à 2011), le Professeur Eduardo Blasco Ferrer, titulaire de la chaire de Linguistique sarde à l'Université de Cagliari. La somme de cette recherche est l'ouvrage, remarquable en tous points, publié à Berlin par les Editions De Gruyter: 163 pages de texte en italien à l'impression serrée mais aérée et très lisible, notes en bas de page, Bibliographie considérable (p. 163-190), 4 Index (p. 191-226): Auteurs, Matières, Onomastique et toponomastique (successivement: "paléosarde », "basque », " ibérique », « autres langues », « radicaux et suffixes paléosardes » classés selon leur origine ou référence linguistique), Mots (successivement: « sarde », « basque », « latin », « autres langues »). Au texte et aux index s'ajoutent les Cartes linguistiques: les territoires actuels de langue basque sont représentés de manière schématique à la page 229 (la région de Bayonne, pour le moins largement bilingue, est située assez loin de la « frontière linguistique » supposée); les cartes de la Sardaigne, accompagnées de notes explicatives, montrent clairement les territoires orientaux de l'île (opposés à la face méditerranéenne occidentale tournée vers la France et l'Espagne) 
où se concentrent les noms de lieux objets de l'analyse et leurs aires de dispersion dans le reste du pays. Enfin une série de photographies en noir et blanc fait voir des lieux au nom restitué en paléosarde, suivi du nom actuel entre parenthèses, qui sonneront plus ou moins familièrement à nos oreilles (selon le degré de restitution à laquelle E. Blasco Ferrer a procédé méthodiquement dans son ouvrage): Aranaké, Desunele, Makumele, Otziddai, Navarrese, Susune, Tuturki. L'ouvrage accorde une place très importante, entre bien d'autres références, aux nombreux composants lexicaux (lexèmes et suffixes) basques, soit qu'ils se retrouvent encore en abondance dans la toponomastique et le lexique de nos régions, soit qu'ils ont pu être rapportés à un «paléobasque » des temps néolithiques obtenu par la méthode de la reconstruction.

La toponymie de la Sardaigne avait depuis longtemps intrigué les spécialistes en étymologie et toponomastique méditerranéennes, italiens avant tout comme il se doit. Certains avaient soupçonné ou perçu des correspondances avec le monde linguistique de l'Hispania antique: l'ibère, langue perdue au début de notre ère et encore aujourd'hui, malgré des progrès considérables, imparfaitement comprise, et le basque, lui maintenu à travers les siècles et les millénaires, tout en reculant et s'altérant sans cesse devant la poussée latinisante et romanisante. Il manquait, comme le dit E. Blasco Ferrer, « la clef » qui permettrait de comprendre la formation de ces noms de lieux « énigmatiques » collectées dans la zone considérée, et d'ouvrir en grand les portes sur le très lointain passé linguistique de la Sardaigne.

Les périodes ainsi balisées et résumées dans un tableau repris dans les « Conclusions et desiderata » qui terminent l'ouvrage (p. 162) vont du " prémésolithique » (IXème millénaire avant notre ère), en passant par le « mésolithique » et le " néolithique » (du IXème au IIIème millénaire), jusqu'au « calcholitique-nuragique » (du IIlème au ler millénaire: soit plus de 3000 ans avant nous). Il y a de quoi faire rêver des bascologues pour qui la documentation linguistique écrite ne remonte guère au delà des derniers temps de l'Antiquité et du Haut Moyen Age et qui savent que, pour une langue reconnue comme "stable», il convient de réfléchir, selon l'espression des historiens, sur le « long temps ». Larchéologie a permis de définir des traits caractérisiques d'une culture spécifique que l'on nomme en Sardaigne «nuragique », répartie en trois « ères » successives couvrant l'âge de bronze: " archaïque » (1800-1600), « moyen » (1600-900), tardif (900-500) (p.42). De ces modes de vie en évolution, société sans villes répartie d'abords en groupes de type tribal, installée en habitats d'environ 7000 nuraghes (la base nur compte «parmi les plus productives et particulières de la toponomastique régionale »), situées « en hauteur et près de cours d'eau » (p. 108), avec sépultures collectives, culte d'une déesse mère progressivement remplacée au nuragique moyen par les divinités masculines amenées de l'extérieur, il reste suffisamment de vestiges en pleine période de christianisation pour que Grégoire le Grand au Vlème siècle puisse décrire et dénoncer les habitants de la Barbagia en ces termes: "Tous les Barbariciens vivent comme des animaux dépourvus de raison, ils ignorent le vrai Dieu, et adorent même des bois et des pierres»(p. 41). N'étaient l'époque, plus tardive, l'auteur et la référence chrétienne, on pourrait penser aux cultes évoqués dans les inscriptions antiques d'Aquitaine : on sait que, dans leur contexte latin, elles présentent tout un lexique où les spécialistes (de Luchaire à Gorrochategui) ont constamment reconnu des éléments d'un « proto-basque » aquitain. Les résultats de l'analyse linguistique coïncident même avec les données, peu discutées, les plus récentes de la génétique, ce qui permet à 
l'auteur de conclure au peuplement de la Sardaigne centrale et montagneuse à la suite « d'une ou plusieurs migrations prénéolithiques de colonisateurs provenant de l'antique Ibérie, et plus particulièrement de l'aire proto-basque dans le nord-ouest de l'Espagne et le sud-ouest de la France (Aquitaine)» (p. 45-47).

La langue parlée dans la Sardaigne pré-sémitique et pré-latine était de la même famille, justement, que la langue basque ou plus précisément sa forme antique dite « paléobasque » et l'ibérique. Après avoir rappelé et soumis à une critique argumentée et rigoureuse les approches et méthodes (extralinguistiques comprises) d'explication jusque-là utilisées, l'auteur démontre que, par les traits structurels et typologiques préalablement définis, la langue de substrat dans laquelle ont été formés les microtoponymes sardes analysés ne correspond pas à une langue de type «flexionnel» ou « fusionnel» comme le sont les langues de la famille dite " indo-européenne » et par exemple le latin, mais à une des langues dites "agglutinantes » ou « modulaires » comme le basque et son ancêtre reconstruit le " paléobasque », l'ibère, l'étrusque, le minoen, le turc etc. (p. 32-35, p. 97). Dans ces dernières à la différence des autres, les marques « relationnelles » (les divers suffixes de déclinaison) s'ajoutent simplement au nom sans le modifier, et de même dans une certaine mesure les éléments de composition. La typologie syntaxique se définit aussi par l'ordre des mots dans la phrase ou le segment de phrase (complétés et compléments), ou encore, pour les langues de type agglutinant, par l'emploi d'une marque unique de nombre et de fonction à la fin du syntagme (non concordance), par l'absence de préfixes et au contraire l'extension de la suffixation. La typologie phonétique présente de même plusieurs traits significatifs communs au paléobasque (dont le basque historique, sur des points essentiels, semble être resté assez proche) et au paléosarde: même système vocalique, structure syllabique prédominante CVC (consonne-voyelle-consonne), absence de p- initial.

Observant l'état actuel de la dialectographie sarde, au chapitre 7 en fin d'ouvrage (p.153157), l'auteur rassemble un ensemble de faits du dialecte sarde « néo-latin dans la Barbagia et les aires orientales (...) qui coincident avec des traits typiques du basque »: l'accentuation, l'alternance vocalique, la perte de $\mathrm{f}$ initial latin (comme en basque ancien, cette consonne est absente aussi en ibérique) et sa résolution par l'aspiration encore notée en sarde à la fin du Moyen Age, maintien des occlusives sourdes intervocaliques, prononciation « renforcée » des vibrantes intiales et, dans certaines régions, prothèse vocalique (on a ainsi du latin rivum le sarde dialectal erriu comme du latin ripam le basque erripa ...). On peut noter qu'il n'est pas fait mention dans l'ouvrage d'un autre trait phonétique extrêmement caractéristique à la fois du basque et de l'ibère: c'est l'absence du groupe consonantique muta cum liquida et son adaptation au basque dans les emprunts latino-romans par l'introduction d'une voyelle d'anaptyx jusqu'à une époque très récente (l'exemple-type est le latin tardif cruce (krutze) > kurutze/gurutze).

La liste des bases toponymiques communes à la toponymie (souvent relevée dans la tradition orale qui survit, comme le fait remarquer l'auteur) des régions centro-orientales de la Sardaigne et à la toponymie basque anciennement documentée et encore en usage pour l'essentiel a de quoi surprendre et impressionner. Au chapitre 5 (« Paleosardo, Paleobasco, Iberico » p. 89-135), après la comparaison des 3 langues au plan typologique, l'auteur liste et commente les radicaux et suffixes que la « confrontation » avec le paléosarde conservé dans 
les toponymes montre comme appartenant au paléobasque, à l'ibère, et « d'origine incertaine ou inconnue ». Ces derniers, "qui ont été alternativement rattachés à un fond paléo-indoeuropéen ou péri-indoeuropéen » mais susceptibles néanmoins d'être étymologisés, au nombre de 17, comptent des termes très employés aussi dans la toponymie historique basque et présents, sous une forme identique ou évoluée, dans le lexique, tels par exemple l'oronyme *karra ( qui se réalise garr(-) en toponymie basque et aurait été le radical de harri " pierre »), gava, nava, *ore ou *sara. Dans les 10 éléments rattachés à l'ibère on relève de même des éléments familiers au basque ou proches des formes basques modernes, parmi lesquels le très connu ili ( en basque médiéval iri « habitat, ville», d'où est issu plus tard le moderne hiri « cité, ville »), mais aussi bel(es)/meles (en basque bele " corbeau », beltz " noir », en sarde il se réalise aussi avec variation de nasale nele ), arki, bar, ortu, lako, turki/urki, dont les correspondances sémantiques ne sont pas forcément proches dans les homonymes ou paronymes basques. Ces références ouvrent sur la comparaison de la toponymie ibère et basque ou " protobasque » (aquitain antique), vaste chantier qui n'en est encore qu'à ses débuts.

Dans la « confrontation » avec le «paléobasque », E. Blasco Ferrer a relevé dans la toponymie sarde 3 suffixes ou séries de suffixes : -ake accompagné d'une longue note de commentaire et discussion serrée pour le rapprochement avec le suffixe locatif et strictement toponymique basque -aga et son étymologie (p. 116, note 95), les suffixes à « diphtongue décroissante »-ai, $-e i,-o i$, et - $t z$. Mais c'est une bonne trentaine de bases lexicales «paléobasques » que l'auteur a reconnue en toponymie sarde, la plupart étant des composants très communs des noms basques de lieu et du lexique toujours en usage : al (h)a " pâture », (h)aran « vallée », ardi « brebis » et ses dérivés toponymiques (le plus commun devait être artola littéralement « cabane à brebis " pour « bergerie »- comme itola « cabane à bœufs » - qui ne semble pas avoir été relevé en Sardaigne), (h)artza (qui est un oronyme et pas le nom de «l'ours »), baso « forêt, bois » (en composition basque basa-) que le sarde a sous la forme masa- (la nasalisation est ordinaire aussi en basque ), berri « nouveau » et « terre nouvellement cultivée », bide « chemin », ertz « bord, orée », gorri « rouge », (h)iri « habitat, ville », iztil « eau stagnante et boueuse », iz " eau, liquide, rosée » reconnu en toponymie médiévale basque dans izotz " gelée blanche » (littéralement « rosée froide» : le mixain Içozta cité en 1350 a un correspondant toponymique sarde curieusement proche Itzotzake), lats « ruisseau, petit cours d'eau » (et voici encore une Funtana di Latzakké sarde bien proche de nos Latsaga, p. 107), logi « fange » qui se réalise lohi en basque historique, lur « terre » (Lutzurró sarde «terre blanche» avec une formation évoluée mais proche du composé basque lu(r)zuri, et la même finale que Luro/Lurro médiéval), mando « mulet », ola « cabane », ona « colline », orri (on est bien près aussi de orre " genévrier » qui n'est pas mentionné pour la Sardaigne) et osto «feuille, feuillage », (h)otz «froid », (h)obi « fosse » (du moins dans le sens clairement donné par la traduction romane médiévale du mot basque, tenu traditionnellment pour un emprunt, étymologie, écrit E. Blasco Ferrer, « que la seule présence du mot en Sardaigne exclut automatiquement », p. 112, note 78), soro « champ libre, pré » rapproché d'une base sor des inscriptions ibériques (et dont l'auteur récuse aussi « pour de claires raisons géolinguistiques et de chronologie » l'étymologie latine généralement proposée, p. 113), *susune présent en toponymie basque sous diverses formes comme zuzun " peuplier tremble», (i)turri « source», avec une voyelle initiale entre parenthèses et selon l'auteur "étymologique comme celle de i-bai fleuve » (alors que la toponymie médiévale basque et antique ibère ne donnent clairement que bai à peu près sans exception; par ailleurs 
it(h)urri paraît difficilement détachable du lexème basque ita/ita- " goutte »), documenté en abondance turri hors du domaine basque ( mais jamais en domaine basque historique, quoique l'aphérèse soit très courante dans la prononciation locale et quotidienne : " $\mathrm{t}(\mathrm{h})$ urria, tturria »), ur « eau » de grande extension en toponymie fort loin des territoires de langue basque (Gard, Drôme ), zuri « blanc » en Sardaigne dans Monte zuri, Riu zuri dans des composés en fait «bilingues » et plusieurs autres.

Quelques-unes des bases « paléobasques » obtenues par reconstruction ne trouvent pas de telles correspondances directes, ni dans la toponymie basque historique ni dans le lexique. Ainsi: *des (p. 103) serait une forme ancienne de leze « gouffre, caverne » conservée seulement par la toponymie sarde dans Desunele (en lexique basque historique les dentales initiales, généralement sonores, sont d'emprunt, sauf dans la conjugaison au présent de 3ème personne, et la latérale remplace parfois une dentale initiale d'emprunt sourde ou sonore: par exemple teca > leka « gousse », danger > lanjer etc.); *dol serait la réduction d'une forme paléobasque à redoublement syllabique (le redoublement syllabique est très banal dans le langage expressif et enfantin, particulièrement en basque moderne) ayant donné en basque historique odol «sang», reconnue dans des noms sardes comme Dolai, Dodoliai (bien que les lexiques divers ne manquent pas de « sanguinaires » botaniques ou autres, le terme historique odol ne semble pas avoir laissé de toponymes basques); *goni (Goni nom de commune sarde) est rapporté à deux mots basques au sens de " hauteur »: gain, que son vocalisme rapproche plutôt de son synonyme garai, tous deux également productifs en toponymie basque, et goi, la nasale sarde rappelant tout de suite son superlatif goien qui n'est pas moins répandu; *nur (pour son extension en toponymie sarde et le rapport sémantique avec l'idée de « construction » voir cidessus), dont on peut se demander s'il n'a pas, plutôt qu'avec le dialectal basque (h)uri variante à voyelle initiale labialisée et dialectalement bien localisée du très commun iri (p. 109-110), quelque rapport avec la base mur(r) (qui ne peut être dans ce cas un héritier du latin muru) également présent en toponymie basque et ibère (l'extension sur ces deux aires linguistiques de Murgi est très remarquable).

Au cours de l'ouvrage, à la fin du même chapitre consacrée à la " taxonomie sémantique » (p. 133-134), dans l'index des noms « paléosardes » (p. 198-208), le lecteur familier d'onomastique et toponomastique basques reconnaîtra d'autres curieuses coïncidences: par exemple Aritzo qui serait, sur haritz « chêne, une formation parallèle au très répandu Hariztoi de nos régions; Baradce (en basque baratze ne peut être compris en toponymie médiévale, où le mot abonde, qu'au sens de " jardin, potager »); Gaillura (il serait rapporté à une base archaïque *gald- ou *gand- qui est peut-être dans le lexique basque dans gandorr « crête, cime » et en tout cas apparemment dans le toponyme médiéval gandaratz/ganderatz cité à partir de 1249); dans les noms d'animaux celui du « renard » azeri, dont l'auteur discute l'étymologie latine généralement admise (p. 133-134, note 167). Et on est un peu étonné - mais il y a peutêtre une bonne raison - que celui du «loup» otso n'ait pas laissé en Sardaigne quelque chose de l'incroyable vogue qu'il a eu en onomastique basque...

De la «théorie sur les origines linguistiques de la Sardaigne néolithique » présentée par le Professeur Eduardo Blasco Ferrer, thèse « innovatrice fondée sur une recherche pionnière » comme il l'écrit (p. 159), les remarques qui précèdent ne peuvent donner qu'une idée bien insuffisante, et il faut en prendre toute la mesure par une lecture complète. Elle révèle encore une fois l'intérêt d'une prospection et d'une analyse méthodiques des toponymies régionales (la 
connaissance de la langue basque historique au-delà des grands textes du XVIe siècle leur doit à peu près tout), et tout spécialement des « microtoponymes plus fidèles à une transmission populaire » (p. 147 note 29), qui peuvent avoir mieux conservé des traces linguistiques très anciennes dans les territoires où les langues locales ont été effacées par les langues dominantes. Le progrès dans la connaissance de l'histoire linguistique c'est aussi un progrès dans l'histoire des peuplements.

La concordance entre les données de l'archéologie (échange de céramique, commerce de l'obsidienne) et les correspondances linguistiques bien établies désormais entre la Sardaigne et l'Ibérie occidentale et principalement les terres ou les peuples de langue basque pose évidemment quelques questions. La première, et sans doute la plus difficile, c'est de trouver une solution historique à cet état de fait : faut-il, comme le propose l'auteur, selon le mode de relations par conquêtes et «colonisations » qui a fait l'histoire des régions méditerranéennes de l'Antiquité aux temps modernes, ou de simples «migrations » de peuples, penser à un mouvement d'expansion de l'Ibérie occidentale vers la Sardaigne orientale ? Ou bien la situation bien visible sur la carte des terres aujourd'hui bascophones à l'extrémité pyrénéenne occidentale d'une part, et des toponymes sardes bascoïdes concentrés au contraire dans l'espace insulaire oriental de l'autre, signalerait-elle des ilôts où le vieux fond linguistique nonindoeuropéeen a pu, à mesures diverses et jusqu'à des époques bien différentes selon les lieux, résister au mouvement général des langues et des civilisations dites « indo-européennes »?

Le questionnement touche du reste le basque lui-même et les bascologues. E. Blasco Ferrer aurait sans doute pu avancer sur un terrain plus aisé si, au moment de développer sa recherche, la connaissance du passé de la langue basque avait été mieux établie, en particulier au plan des étymologies, comme il le signale avec raison: "Divers parallélismes étroits, de caractère formel référentiel, entre radicaux basques, tenus pour des emprunts ou d'origine controversée, et radicaux paléosardes, doivent conduire maintenant avec un peu plus d'intensité les spécialistes de basque à chercher des étymologies internes à leur langue (...)» (p. 137 note 3). On peut ajouter aussi des concordances et donc des voies d'explication étymologique hors des emprunts latino-romans - «à l'extérieur » de la langue elle-même, qui n'a pas pu, par on ne sait quel « miracle» inédit, naître et se développer toute seule in situ. Il y a certes des chercheurs engagés dans cette voie, non moins difficile que celle de la définition du (ou des) «substrat(s) » sarde(s) dans laquelle le Professeur E. Blasco Ferrer s'est avancé. Mais on attend toujours un Dictionnaire étymologique basque fiable et suffisamment argumenté, qui ne sera sans doute qu'une étape, on l'espère décisive, dans une entreprise appelée peut-être à rester à quelque titre imparfaite. Il en est ainsi pour toute démarche scientifique, surtout dans les domaines inexplorés, ou peu et mal explorés. La fameuse formule latine que l'auteur place dans les « Desiderata » qui terminent son ouvrage vaut pour tous les domaines de recherche : citius emergit veritas ex errore quam ex confusione "la vérité émerge plus vite de l'erreur que de la confusion ». Si toute la vérité n'est peut-être pas dite - l'est-elle jamais dans certains domaines? -, l'exploration menée par l'auteur donne l'impression d'avoir été suffisamment et méthodiquement balisée et argumentée pour être désormais un outil dont l'efficacité dépasse de loin les limites territoriales qu'il lui a assignées. 